\title{
Engaging the Public in Planning for Disaster Recovery
}

Jennifer Horney - Corresponding Author

Associate Professor, Epidemiology and Biostatistics

Department of Epidemiology and Biostatistics

Texas A\&M Health Science Center School of Public Health

1266 TAMU

College Station, TX 77843

horney@srph.tamhsc.edu

979-436-9391

Mai Nguyen

Associate Professor, City and Regional Planning

University of North Carolina at Chapel Hill

CB\# 3140

Chapel Hill, NC 27599

mai@unc.edu

David Salvesen

Research Associate, Institute for the Environment

University of North Carolina at Chapel Hill

CB\# 1105

Chapel Hill, NC 27599

dsalv@email.unc.edu

Olivia Tomasco

Research Assistant, Sociology

Texas A\&M University

4351 TAMU

College Station, TX 77843

otomasco@tamu.edu

Philip Berke

Professor and Director

Department of Landscape Architecture \& Urban Planning, Institute for Sustainable Coastal Communities

College of Architecture, Texas A\&M University

3137 TAMU

College Station, TX 77843

pberke@arch.tamu.edu 
Acknowledgements: This work was supported by the National Science Foundation (NSF), Award CMMI-1066310. The findings and conclusions are those of the authors and do not necessarily represent the official position of NSF. No authors have any financial interest or benefit arising from the direct applications of this research. 


\begin{abstract}
Communities engage in various ways with stakeholders around plan development. This project aims to validate quantitative content analysis scores for participation in disaster recovery plans with follow-up key informant interviews. Recovery plans from 87 counties and municipalities adjacent to the U.S. Atlantic and Gulf Coast were collected and content analyzed using a plan coding protocol. Four jurisdictions - two with high and two with low scores in the plan quality principle of participation - were selected for follow-up key informant interviews.

Several themes emerged from the qualitative data. Public engagement in recovery planning is more successful when planners actively engage individuals and groups and when dedicated staff are assigned to participation activities. While addressing the needs of socially and physically vulnerable residents can be challenging, there are effective ways of encouraging their participation. While the sample size of this study was small and the findings may not be generalizable to areas outside of the U.S. Atlantic and Gulf Coasts, findings do support the planning research literatures' suggestion that increased participation is associated with higher plan quality. Our findings provide specific examples for planners interested in increasing participation. However, an unanswered question remains as to the extent to which increased engagement in recovery planning will lead to increased stakeholder awareness of risk, available resources, and support for policies that build resilience.
\end{abstract}

Keywords: disaster recovery; planning; participation; vulnerability 


\section{Engaging the Public in Planning for Disaster Recovery}

\section{Introduction}

Over the last several decades, the planning literature has come to the consensus that stakeholder participation in the planning process can improve the quality of plans. The recognition of the importance of participation to both plan quality and the fidelity of implementation is related to many factors, including the democratization of environmental decision-making, an increased knowledge in citizen science, and policy trends that emphasize partnership (Reed 2008). This change in emphasis has also been supported by the evolution of planning theory, with an increased focus on the role of the planner as a communicator, an intermediary among stakeholders, and a consensus builder (Fainstein 2000).

Much of the published research has focused on the importance of participation in the successful development and implementation of comprehensive, hazard mitigation, and land use plans (Brody, Godschalk and Burby 2003; Brody 2003). For example, in 2003, Burby tested the hypothesis that involving a "broad spectrum of stakeholders in the plan making process" would improve the quality of comprehensive plans and concluded that stakeholder involvement can make plans better (pp. 34). In a review of Australian and U.S. research, Pearce (2003) concluded that sustainable hazard mitigation requires public participation and community-based planning, while in a study of land use planning, Burby and colleagues (2000) concluded that "community agreement over a mitigation approach must be built on a foundation of public support" (pp. 100). Few studies concentrating on the role of participation in the quality of pre-disaster recovery plans have been published. Pre-disaster recovery plans developed without stakeholder participation may fail to adequately include local knowledge and capacities, shortchanging local residents and 
complicating pre-disaster recovery plan implementation after disasters (Olshansky, Hopkins and Johnson 2012; Phillips 2009).

In concept, the process of developing a plan can, in and of itself, help create, strengthen, and engage members of the general public as well as networks of various stakeholder groups (e.g., affordable housing organizations, churches, chamber of commerce) (Berke et al. 2010). For example, participation in and awareness of the process of developing a plan can improve disaster outcomes and future resiliency because planning is where community members set priorities and make choices that affect their future vulnerability, as well as that of the built and natural environment (Manyena 2006; Olshansky and Johnson 2010). Previous research has found significant correlations between participation in hazard mitigation planning and planners' beliefs, choices and behaviors (Stevens, Berke and Song 2009). When planners pursue more participatory objectives, such as "fostering citizen influence in hazard mitigation," the resulting hazard mitigation plans have $76 \%$ more mitigation measures when compared with jurisdictions that do not include participatory objectives (Burby 2001). Engagement may also have additional benefits if it leads to socially vulnerable groups (e.g., members of racial/ethnic minorities, the elderly, poor and persons living with disabilities) becoming more likely to be aware of and benefit from government programs intended to mitigate risk (Ojerio et al. 2011; Pearce 2003).

However, important challenges to effective participation in planning have also been identified. Funding for the number of planners necessary to engage and provide support to local residents may not be available (Sirianni 2007). Staff may not know how to carry out an effective participation program and may not have received training on the types of proactive efforts 
needed to directly involve residents, beyond traditional groups such as developers and neighborhood groups, in planning (Burby 2001; Pearce 2003). Residents may be apathetic to the planning process, may lack the education and resources to engage effectively, or may represent only their own (or their organization's) self-interest (Derkzen, Franklin and Bock 2008; Herbert 2005).

While the methods and content analysis protocols for normative plan quality evaluation are wellestablished in the plan quality literature in general (Lyles and Stevens 2014), and in studies of plan quality and participation specifically (Burby 2003), a mixed-methods approach that includes both quantitative and qualitative data may help validate the quantitative content analysis approach. A mixed-methods approach may also better address some plan weaknesses by enabling the collection of best practices and promoting effective interventions that can be widely adopted to improve plans. One way of collecting this type of information is through conducting key informant interviews (Kumar, Stern and Anderson 1993; Coburn 2003; Wridt 2010). When combined with a code-based plan quality analysis, key informant interviews can assist with gauging more subjective perceptions about the recovery plan and the planning process.

In this project discussed below, data collected as part of qualitative interviews are used to show how participation may improve plan quality and identify the factors that lead to increased participation to improve our understanding of how and why enhanced public participation could lead to better plan quality. The mixed-methods approach used here - combining the quantitative metrics obtained through a recovery plan content analysis and survey with the qualitative data obtained through the key informant interviews - attempts to address the "complex, multi- 
dimensional, nonlinear nature of disaster recovery" and better understand the ways in which researchers can quantitatively and qualitatively assess a community’s recovery plan, and ultimately, their recovery and resilience to future disasters (Johnson and Hayashi 2012 p.217).

\section{Methods}

\section{Data Sources and Sample Selection}

The initial sampling frame for the study included coastal counties $(n=107)$ and coastal municipalities $(n=175)$ with at least 10,000 residents along the Southeastern Atlantic and Gulf Coasts from Virginia to Louisiana. Of these, 87 (49.7\%) counties and municipalities had some type of recovery plan that was publically available online. To be included in the sample, recovery plans were required to meet at least two of the following three criteria: include a vision statement or goals, a fact base that identifies the hazards present, and recovery policies designed to achieve the vision or goals. Recovery plans could be stand-alone $(n=9)$ or included as an element in a local comprehensive plan $(n=35)$ or other type of plan, such as an emergency management $(n=40)$, local mitigation strategy $(n=1)$, or hurricane plan $(n=1)$. All plans included were adopted or amended between 2007 and 2012.

The 87 recovery plans were content analyzed by the research team to assess how well the seven plan quality principles - goals, fact base, policies, inter-organizational coordination, participation, implementation, and monitoring and evaluation - were accounted for in each recovery plan (Berke et al. 2014). In addition, the lead public official responsible for the administration of each of the 87 jurisdictions' recovery programs was invited to complete an online survey related to the recovery plan. Fifty-five of the 87 (63\%) county and municipality 
officials completed the survey (Horney et al. 2016).

From the 55 jurisdictions with both plan quality and survey data, we selected four jurisdictions for the key informant interviews; two with a participation score greater than the overall mean (0.16: Range 0-1) and two with a participation score below the overall mean. A modified snowball technique was used to identify key informants in each of the four jurisdictions. Starting with the local planner or emergency management official who completed the online survey, the research team asked for referrals to develop a list of up to 5 contacts for each community, including planners, emergency managers, and elected officials. Interviews were scheduled by email or telephone and informed oral consent was obtained.

\section{Interview Guide}

A written interview guide consisting of twelve closed- and open-ended questions was developed and used to guide the semi-structured interviews. Informants were asked to address several issues related to the jurisdiction's recovery plan and the planning process, including questions about the extent of and barriers to public participation in plan development and the inclusion of vulnerable populations. Additional questions asked about the implementation of the jurisdiction's recovery plan, as well as its integration with other adopted plans, such as comprehensive, land use, or capital improvement plans. Informants were asked to list ways in which the recovery plan made their jurisdiction less vulnerable to disasters. Finally, informants were asked to refer the interviewer to others in their jurisdiction who might be able to answer questions about the recovery plan and planning process. All interviews were recorded, transcribed, and analyzed in Microsoft Excel (2010) to determine themes using inductive or open coding (i.e., themes are not 
predetermined, but rather emerge from data through examination and comparison).

All materials were reviewed and determined to be exempt by the Institutional Review Board at the University of North Carolina at Chapel Hill (\#13-2292).

\section{Results}

Research team members completed interviews with between two and three representatives from each of the four counties in the sub-sample, including counties in Florida, North Carolina, and South Carolina, during September $2014(\mathrm{n}=10)$.

Of the four counties where the key informant interviews were conducted, three had recovery plan elements that were included in an emergency management plan, including both of the plans that scored highly in the participation principle. The other plan was a recovery plan included as an element of a comprehensive plan, which has been demonstrated in a prior study to be associated with lower participation, perhaps due to their focus on a wide range of development issues, only one of which is recovery (Berke et al. 2014). In terms of overall plan quality, all the plans in the prior study of 87 generally offered a weak framework to guide recovery decisions and achieve long-term resiliency (Berke et al. 2014). However, those scoring higher than average in participation had lower item scores in every other principle except organizational coordination.

There were some differences in the 4 selected jurisdiction's capacity for participation in recovery planning as reported in the online survey of emergency managers / lead planners. In the two counties with a higher than average participation score for their recovery plan, more staff time was assigned to participation ( $0.50 \mathrm{FTE}$ and $1.00 \mathrm{FTE}$ ) as compared to counties with lower 
participation scores ( 0.25 FTE and 0.33 FTE). However, all counties began participation activities in the initial, pre-planning phase of the recovery plan development. Both higher scoring counties identified gaining public support for the recovery plan as the most important reason for incorporating public participation into plan development, while lower scoring counties felt that the most important reason for participation was to educate the public. There was little difference in the number of groups targeted for participation by high scoring ( 8 and 10 of 13 groups) and low scoring (6 and 10 of 13 groups) counties. However, the level and type of participation reported was significantly different, with high scoring counties being significantly more likely to report "extensive" participation by a larger number of groups $(\mathrm{P}(\mathrm{T} \leq \mathrm{t}<0.001))$. Higher scoring counties used fewer techniques to recruit groups to participate ( 1 and 3 of 12 techniques for high scoring counties, 4 of 12 techniques for both low scoring counties); however, they relied on different types of participation opportunities, including focus groups and key informant interviews, while lower scoring counties relied exclusively on public hearings and community forums. As result, the number of individual participants was higher in the high scoring counties (75 and 200 participants) versus lower scoring counties (25 and 40 participants).

Three major, interrelated themes emerged from the key informant interviews, pertaining to the importance of 1) using active techniques to engage the public; 2) assigning dedicated staff to recovery planning; and 3) specifically addressing the needs of socially and physically vulnerable populations in your recovery planning, as well as their participation in the recovery plan development process. Each theme, supported by specific participant responses is discussed in detail in the following section. 


\section{Active engagement is critical for meaningful participation}

In the two counties with higher participation scores, key informants identified active techniques for encouraging participation, including surveying the public, working one-on-one and in small groups, and utilizing established partnerships with business and places of worship. As one respondent put it, "I just have to go out in the community... and speaking together with a community member means that the audience will be more receptive." Faith-based groups, community organizations, neighborhood groups, and business groups were all cited as examples of potential trusted partners. The number of opportunities to participate matters too. One respondent mentioned having nearly 100 meetings within the county in order to make them accessible to all the neighborhoods. A planner or emergency manager's knowledge of their communities is essential for active engagement. As one respondent put it "understanding the demographics and the needs of the population" will help ensure participation, by knowing when and where residents can attend meetings and when meetings should be held in another language. Nearly all key informants recognized that true engagement isn't easy. "It's extremely difficult to involve the public in meaningful ways," one key informant pointed out; however, "local knowledge can be very relevant" particularly in the actual recovery process.

In the two counties with lower participation scores, the engagement of the public relied more on passive measures, such as public comment periods and posted notices on websites and in the newspaper. In one county, a limited number of public meeting were held, with "only about 6 or 7 people attending, including representatives from the State, County, and FEMA, and one time, a city councilman." Overall, attendance at meetings was limited to county agencies and other governmental officials, rather than groups more wholly external to the planning process and all 
meeting were reportedly held at governmental buildings or facilities. Several respondents mentioned the use of press releases sent to the local newspaper as a means of recruiting members of the public to attend meetings, but commented that "the public usually does not attend or become engaged in those types of meetings." One respondent pointed out the importance of shifting towards the use of social media, such as Facebook, as well as websites for community outreach, to reach a broader group of residents who are likely not receiving information via traditional printed media.

Assigning dedicated, specialized staff to recovery can help ensure the attention needed for success

Both of the counties with high participation scores pointed to the importance of assigning dedicated staff to recovery. As one respondent put it, "In the end, our goal is to build a more disaster resilient community, and you do that 365 days a year." Another respondent pointed out that "taking a planner and dedicating them to recovery" was necessary in order to prevent the constant pressure to focus on response. Another pointed out that having a staff member who specialized in recovery allowed for the implementation of the recovery plan even after small or isolated disasters such as localized flooding or a building collapse. This ability to implement the plan in response to smaller emergencies provides opportunities to "talk to liaisons within those communities and collaborate, even if it's on a small scale." Having a staff member assigned to recovery can also help with finding the time necessary to build strong relationships, particularly with community leaders, faith-based groups, and groups representing vulnerable populations before a disaster. Dedicated staff have time to hold meetings during "off hours" that are most optimal for getting a diverse group of residents to participate, as well as time to dedicate to 
occasionally attending regularly scheduled meetings of community groups. "Even knowing each other a little beforehand makes a tremendous difference in the working relationship needed during the highest stressed times within the community recovery."

Neither of the counties with low participation scores mentioned assigning staff specifically to recovery planning. However, several barriers that were mentioned by respondents from these two counties might be addressed by assigning more staff time to recovery planning, including public apathy towards recovery planning and the engagement of minority groups (Hispanics and religious minority groups were specifically reported) who lack trusting relationships with governmental authorities and subsequently "avoid contact" with them. Relationship building with these groups could be modeled on successful engagement of other groups, including the elderly, the marine / fishing industry, and hospitals and health departments that were reported by key informants.

Social and physical vulnerability must be specifically addressed in recovery planning, in part through the participation of vulnerable individuals and groups who represent them

Counties with high participation scores reported "gaining a better perspective on communities with the greatest needs...by partnering with these groups within the communities." Again, they leveraged faith-based groups, known community leaders, as well as the leaders of agencies that represent vulnerable populations in order to "actively work to close the gaps" in recovery planning for vulnerable populations. In addition to county agencies, other social service groups that fall under a Recovery Support Function's key areas for assistance "have advocacy focused 
staff that being the perspective of vulnerable people to the table at all times." Respondents reported challenges in planning for vulnerable populations due to changing Federal guidance on this issue (e.g. FEMA's Comprehensive Preparedness Guide) and resistance from certain vulnerable groups, such as the elderly, to heed warnings or register for automated disaster messaging through text message or email. However, a consistent focus on "a vision for long-term community recovery... where the next phase of development can be improved," may help these counties in their focus on reducing vulnerability and increasing resilience in the long run. While counties with lower participation scores did not have success in engaging vulnerable individuals or groups directly in the planning process, they did utilize other tools in an effort to reflect the specific concerns of these groups in their plans. One respondent pointed out that their office utilized geographic information systems software "to overlay information (about vulnerable groups) with U.S. Census data and hazard exposures such as the 100 year flood plain and storm surge inundation zones in order to identity where their vulnerabilities really were." They also reported on efforts to leverage existing relationships with hospitals and public health to reach out to vulnerable populations, as well as the development of grant applications that would seek funds to mitigation physical vulnerabilities to hazards through elevation or acquisition of properties in the flood plain.

\section{Discussion and Conclusions}

In this small case study, higher scores in the participation plan quality principle on a quantitative recovery plan protocol were associated with differences in the county's choices related to engagement as reported in key informant interviews. Counties with recovery plans with higher participation scores tended to have more staff assigned to participation. In contrast to some of the 
published literature, which cites the challenge of engaging a sometimes apathetic public without subject matter expertise in the technical aspects of planning, planners and emergency managers in these counties saw participation as a two-way street, with benefits for both the public and for planners in the exchange of information and feedback. Realization of these mutual benefits simply seems to require more staff time, which could have important implications for practice in terms of decision-making related to the utilization of scare fiscal and human resources.

Counties with higher quantitative plan quality scores in participation also had more and different types of opportunities for stakeholders to participate in recovery planning, including the use of more intentionally active opportunities like focus groups and household surveys versus more passive public forums. This likely reflects the higher capacity for engagement of these jurisdictions in general, which may help them to engage and connect with more individuals and organizations as part of the disaster recovery planning process, as well as to maintain those connections over time. For example, counties with higher participation also reported engaging in quality improvement initiatives, extensive documentation of baseline measures, ongoing evaluation of what works in terms of engagement, and making improvements when their jurisdiction's plans are updated.

This study, although small, does provide some additional evidence to validate the findings from the plan quality literature, which appear to be reflective of the priorities and activities of planning practitioners. The themes identified in the qualitative data collected in the key informant interviews do seem to support the validity of the recovery plan content analysis protocol. However, they also identify additional areas that may be of value to include on future protocols, 
including separately documenting the participation of governmental groups and public officials from members of the "public." While collaboration around recovery planning through the participation of government departments and elected officials is important, it may be viewed differently than participation by groups more external to the process such as faith-based groups, agencies that represent vulnerable populations, and business groups. Another potential improvement to plan quality protocols may be more direct linkages with recovery planning guidelines, such as FEMA's Self-Help Guide for Long-Term Community Recovery Planning. Without these types of linkages with planning guidelines, it is unlikely that scholarship related to recovery plan quality will be integrated into practice. An important challenge remains to ensure that plan quality protocols can effectively capture the association between improved plan quality and better recovery outcomes, and not simply the ability to effectively address plan guidelines, scorecards, or checklists. 


\section{References}

Fainstein SS (2000) New directions in planning theory. Urban affairs review, 35(4),pp.451-478.

Reed MS (2008) Stakeholder participation for environmental management: A literature review. Biological Conservation, 141(10),pp.2417-2431.

Brody SD, Godschalk DR, Burby RJ (2003) Mandating citizen participation in plan making: Six strategic planning choices. Journal of the American Planning Association, 69(3), pp.245-264.

Brody SD (2003) Are we learning to make better plans? A longitudinal analysis of plan quality associated with natural hazards. Journal of Planning Education and Research, 23(2), pp.191201.

Burby RJ (2003) Making plans that matter: Citizen involvement and government action. Journal of the American Planning Association, 69(1), pp.33-49.

Pearce L (2003) Disaster management and community planning, and public participation: how to achieve sustainable hazard mitigation. Natural hazards, 28(2-3), pp.211-228.

Burby RJ, Deyle RE, Godschalk DR, Olshansky RB (2000) Creating hazard resilient communities through land-use planning. Natural hazards review, 1(2), pp.99-106.

Olshansky R, Hopkins L and Johnson L (2012) Disaster and recovery: Processes compressed in time. Natural Hazards Review, 13(3), pp.173-178.

Phillips B (2009). Disaster recovery. Boca Raton: CRC Press.

Berke P, Cooper J, Salvesen D, Spurlock D and Rausch C (2010) Building capacity for disaster resiliency in six disadvantaged communities. Sustainability, 3(1), pp.1-20.

Manyena S (2006) The concept of resilience revisited. Disasters, 30(4), pp.434-450.

Olshansky R and Johnson L (2010) Clear as mud. Chicago: American Planning Association.

Stevens M, Berke P and Song, Y (2009) Public participation in local government review of development proposals in hazardous locations: Does it matter, and what do local government planners have to do with it? Environmental Management, 45(2), pp.320-335.

Burby RJ (2001) Involving citizens in hazard mitigation planning: Making the right choices Australian Journal of Emergency Management, 16(3), pp. 45-51.

Ojerio R, Moseley C, Lynn K and Bania N (2011) Limited involvement of socially vulnerable populations in Federal programs to mitigate wildfire risk in Arizona. Natural Hazards Review, 12(1), pp.28-36. 
Sirianni C (2007) Neighborhood planning as collaborative democratic design. Journal of the American Planning Association, 73(4), pp.373-387.

Derkzen P, Franklin A and Bock B (2008) Examining power struggles as a signifier of successful partnership working: A case study of partnership dynamics. Journal of Rural Studies, 24(4), pp.458-466.

Herbert S (2005) The trapdoor of community. Annals of the Association of American Geographers, 95(4), pp.850-865.

Lyles W and Stevens M (2014) Plan quality evaluation 1994-2012: Growth and contributions, limitations, and new directions. Journal of Planning Education and Research, 34(4), pp.433-450.

Kumar N, Stern L and Anderson J (1993) Conducting interorganizational research using key informants. Academy of Management Journal, 36(6), pp.1633-1651.

Coburn J (2003) Bringing local knowledge into environmental decision making improving urban planning for communities at risk. Journal of Planning Education and Research, 22(4), pp.420433.

Wridt P (2010) A qualitative GIS approach to mapping urban neighborhoods with children to promote physical activity and child-friendly community planning. Environment and Planning B: Planning and Design, 37(1), pp.129-147.

Johnson LA and Hayashi H (2012) Synthesis efforts in disaster recovery research. International Journal of Mass Emergencies and Disasters, 30(2), pp.212-238.

Berke P, Cooper J, Aminto MM, Grabich S, and Horney J. (2014). Adaptive planning for disaster recovery and resiliency: An evaluation of 87 local recovery plans in eight states. Journal of the American Planning Association, 80(4), pp.310-323.

Horney JA, Spurlock D, Grabich S, Berke P. (2016) Capacity for stakeholder participation in recovery planning. Planning Practice \& Research, 31(1),pp.65-79. 


\section{Author Biographies}

Jennifer Horney is an Associate Professor of Epidemiology and Biostatistics at the Texas A\&M Health Science Center School of Public Health and a Fellow of the Hazard Reduction and Recovery Center at Texas A\&M University. Her research focuses on the impacts of disasters on health and the ability of plans to improve post-disaster outcomes.

Mai Nguyen is an Associate Professor of City and Regional Planning at the University of North Carolina at Chapel Hill. Her research focuses on social and spatial inequality, urban growth, and the relationship between the built and social environment.

David Salvesen is a Research Associate at the Institute for the Environment at the University of North Carolina at Chapel Hill and the Deputy Director of the Center for Sustainable Community Design. His research interested include land use policy and sustainable growth.

Olivia Tomasco is an undergraduate research assistant in the Department of Sociology at Texas A\&M University.

Philip Berke is a professor in the Department of Landscape Architecture and Urban Planning and the Director of the Institute for Sustainable Coastal Communities at Texas A\&M University. His primary research interests are in land use and environmental planning, urban ecology, and community resilience to hazards. 